\title{
ANÁLISIS ESTRUCTURAL Y CRITERIOS DE DISEÑO DE DEPÓSITOS CILÍNDRICOS DE HORMIGÓN PRETENSADO*
}

\author{
(STRUCTURAL ANALYSIS AND DESIGN CRITERIA OF CIRCULAR PRESTRESSED \\ CONCRETE TANKS)
}

Joan Miquel Vilardell Vallès**, Dr. Ingeniero de Caminos, Canales y Puertos

ESPAÑA

Fecha de recepción: 16-XI-95

$681-32$

\begin{abstract}
RESUMEN
El presente trabajo se centra en el estudio del comportamiento estructural de los depósitos cilindricos de hormigón pretensado. Los objetivos perseguidos son, por una parte, el desarrollo de un modelo de análisis conjunto de la estructura (pared-solera-cimentación) capaz de caracterizar con rigor la rigidez de la unión entre la pared y la solera. Por otra parte, y en base a múltiples estudios paramétricos realizados con un programa desarrollado a partir del mencionado modelo, se presentan diferentes métodos de cálculo de los esfuerzos de la pared, para una unión genérica en la base, que generalizan otros métodos simplificados usuales en la literatura técnica; se proponen funciones de pretensado que definen la distribución y la fuerza de pretensado adecuadas para mantener comprimida circunferencialmente la pared cuando el depósito está solicitado por el empuje hidrostático, con una tensión mínima de compresión circunferencial exigida en proyecto; $y$, por último, se proponen criterios para definir el tipo de unión más conveniente en la base, en función de la geometría del depósito y de los criterios de diseño del pretensado.
\end{abstract}

\section{SUMMARY}

The present work is based on the structural behaviour of circular prestressed concrete tanks. In this study, an analytical linear elastic model that considers the soil-tank interaction under axisymmetric loads, taking into account the movement capacity of the wall-to-base connection, is developed. Parametric studies are carried out in order to find the effects on the wall of all parameters governing the stiffness of the wall-to-base connection. Different methods to analyse the main forces of a circular prestressed concrete wall with free edge at the top and a generic wall-to-base connection are presented, which generalize some simplified methods conventionally adopted. A procedure for the determination of a circumferential prestressing function is suggested, in order to ensure a suitable residual compressive stress in the circumferential direction when the tank is full. Furthermore, some criteria about the design of the wall-tobase connection are proposed, taking into account both the tank geometry and the prestressing criteria.

\section{Introducción y objetivos}

Los depósitos de agua son unas estructuras habituales en nuestra geografía, debido a su misión reguladora de caudal $\mathrm{y}$ de presión en las redes de abastecimiento. La actual política hidráulica seguida por varias administraciones indica que en los próximos años se llevará a cabo la construcción de numerosos depósitos con capacidades comprendidas entre los 2.000 y $20.000 \mathrm{~m}^{3}$. Para este rango de volúmenes, una de las soluciones más competitivas

\footnotetext{
(*) Este artículo es un resumen de la tesis doctoral de D. Joan Miquel Vilardell, dirigida por D. Enrique Mirambell y D. Antonio Aguado de Cea, leida en la Escuela Técnica Superior de Ingenieros de Caminos, Canales y Puertos de la Universidad Politécnica de Catalunya en julio de 1994, y que obtuvo la calificación apto "cum laude" por unanimidad.

(**) El autor agradece las ayudas recibidas del Ministerio de Educacióny Ciencia (beca F.P.I) y de la Universitat Politècnica de Catalunya U.P.C durante la realización de su tesis doctoral.
} 
tanto económica como estructuralmente son los depósitos cilíndricos de hormigón pretensado. Éstos se distinguen de otras tipologías por ser capaces de desarrollar dos mecanismos de respuesta a las acciones que los solicitan: mecanismo de deformación anular y mecanismo de deformación por flexión.

La revisión del estado del conocimiento refleja que el número de normas y de publicaciones dedicadas a estas estructuras es muy inferior al correspondiente a otros tipos estructurales, como pueden ser los puentes y los edificios, aspecto que dificulta la tarea del proyectista. En particular, no existen criterios unánimemente aceptados sobre el diseño de la unión entre la pared y la solera ni sobre el diseño del pretensado, dos de los puntos principales que debe abordar el proyectista de un depósito cilíndrico. El tipo de unión en la base del depósito influye significativamente en el comportamiento estructural de la pared, ya que, cuanto menor es la coacción al movimiento, mayor importancia adquiere el mecanismo de deformación anular en el comportamiento estructural del depósito. Ello repercute en los criterios de análisis y de diseño del depósito, y, en consecuencia, en el coste final de la estructura.

Si bien no existe una regla de buena práctica globalmente aceptada, la unión monolítica entre la pared y la solera (continuidad de desplazamiento radial y de giro) es una solución usual para depósitos con capacidades inferiores a $10.000 \mathrm{~m}^{3}$. Las razones que llevan a varios autores a aconsejar este tipo de unión se basan en motivos constructivos (hormigonado continuo de la unión, con la posibilidad de aumentar localmente el espesor de la pared para afrontar con mayor eficacia la flexión), de estabilidad (rigidez ante acciones horizontales), estanquidad (con espesores razonables se controla la fisuración de forma satisfactoria) y mantenimiento.

Sin embargo, uno de los mayores inconvenientes de esta unión es que, a partir de ciertas alturas de pared, la fuerza necesaria de pretensado y los esfuerzos de flexión vertical empiezan a ser importantes. Es por este motivo por lo que, para depósitos de volumen superior a $10.000 \mathrm{~m}^{3}$, la unión entre la pared y la solera suele materializarse con un apoyoflexible (independizando de esta manera parcialmente el desplazamiento radial y el giro entre ambos elementos).

Para reducir el valor de los esfuerzos de flexión y minimizar la fuerza de pretensado cuando la unión es monolítica, mejorando así el comportamiento estructural y ampliando el rango de aplicación de esta tipología, pueden plantearse varias actuaciones. La primera de ellas se centra en la definición de nuevos métodos de cálculo (Van Breugel, 1984; Aguado et al., 1985; Vilardell, 1990) que consideran la rigidez al giro de la unión. La segunda actuación consiste en la optimación de la función de pretensado circunferencial, con el objeto de desarrollar en la medida de lo posible únicamente el mecanismo de deformación anular de la pared, minimizando tanto los esfuerzos de flexión como la fuerza de pretensado necesaria para mantener comprimida la pared durante la vida útil de la estructura (Van Breugel, 1984; Brondum-Nielsen, 1985; Llombart y Antón, 1985; Aguado et al., 1989).

El trabajo desarrollado en la tesis (Vilardell, 1994) se centra en el ámbito de los depósitos cilíndricos de hormigón pretensado, con una unión genérica en la base. Los distintos estudios se han dirigido hacia la consecución de los siguientes objetivos principales:

- Desarrollar un modelo de análisis estático del depósito que contemple la interacción entre la pared, la solera y el terreno de cimentación.

- Implementar el modelo desarrollado en un programa de cálculo, estudiando la idoneidad del mismo mediante contraste con otros métodos numéricos.

- Analizar con el mencionado programa la influencia relativa de las diferentes variables que definen las rigideces de la unión, tanto en el comportamiento estructural del depósito como en la definición del pretensado circunferencial.

- Proponer unas formulaciones para el cálculo de la pared, en base a los estudios paramétricos realizados. Estas formulaciones se basan en métodos simplificados usuales utilizados en proyecto (Girkmann, 1956; Del Pozo, 1967; Ghali, 1979), incluyendo en éstos el efecto de las variables más significativas que definen las rigideces de la unión.

- Definir la función óptima de pretensado, tanto en lo referente a la fuerza necesaria para mantener comprimida la pared con una tensión mínima circunferencial en la hipótesis de depósito lleno, como en lo referente a la forma de la función. Los criterios propuestos tienen en cuenta el tipo de unión en la base.

- Proponer otros criterios de proyecto, que permitan seleccionar el tipo de unión más adecuado en función de la geometría del depósito y del diseño del pretensado.

\section{Desarrollo del trabajo}

Para conseguir los objetivos propuestos se han desarrollado distintos trabajos, los cuales dan contenido a los ocho capítulos en que se fragmenta la tesis.

Inicialmente se centra al lector sobre el tema de estudio, planteándose los antecedentes y la razón de ser de la tesis, los objetivos perseguidos y el método_seguido para su consecución.

Seguidamente, se presenta una revisión exhaustiva del 
estado del conocimiento, distinguiendo principalmente las bases de los distintos modelos de análisis estructural recogidos en la bibliografía consultada, los estudios más relevantes relativos al diseño de la unión pared-solera y los trabajos relativos al diseño del pretensado circunferencial.

A continuación, se desarrollan las bases del modelo propuesto para el análisis de interacción estática suelodepósito, válido para cualquier condición de borde en la unión pared-solera. Este modelo considera un comportamientolineal para los materiales y se basa en una solución analítica desarrollada a partir de la teoría de láminas cilíndricas de revolución y de la teoría de flexión simétrica de una placa circular sobre un medio elástico (Timoshenkoy Woinowsky-Krieger, 1959). Las acciones consideradas presentan simetría de revolución.

El contacto entre el terreno de base y la solera se caracteriza según el modelo discontinuo propuesto por Winkler, si bien el modelo también permite contemplar la hipótesis de indeformabilidad del terreno. Enfunción de la combinación de acciones que se considere, puede existir un despegue de la solera con respecto al terreno, que da lugar a unas condiciones no lineales de contorno. El modelo desarrollado permite contemplar la mencionada no linealidad, con lo que se supera la práctica habitual de no considerar dicho efecto.

El modelo propuesto se ha desarrollado en un programa informático, compatible con un ordenador personal (programa $T A N K$ ), con el que se define, de manera automática, la distribución óptima de pretensado en función de las acciones y del criterio tensional fijados, dimensionándose posteriormente la armadura necesaria para satisfacer los diferentes estados límites últimos y de servicio. La dificultad de contrastar el modelo con resultados experimentales medidos en estructuras reales, ha sugerido acudir a un modelo numérico basado en el método de los elementos finitos, con el que se han contrastado las diferentes hipótesis consideradas.

Con el programa desarrollado se han realizado múltiples estudios paramétricos (divididos en dos grupos independientes) con los que se pretende visualizar y cuantificar la influencia de los principales parámetros de diseño de un depósito en su respuesta estructural, permitiendo así, en función de los resultados obtenidos, dictar criterios de análisis y diseño de fácil aplicación por el proyectista.

El primer grupo de estudios paramétricos analiza la influencia en el comportamiento del depósito de las principales variables que intervienen en la modelización de la unión entre la pared y la solera. Estas variables son el propio tipo de unión diseñada (monolítica o articulada), los espesores de la solera y de la pared, y la rigidez del terreno.
Los estudios realizados han confirmado la sensibilidad del comportamiento estructural de los depósitos cilíndricos a una variación de cualquiera de estas variables. Éste es muy diferente según se considere un apoyo flexible en la base o una unión con el movimiento radial impedido (articulación fija o unión monolítica). En el primer caso, el mecanismo de deformación anular de la pared predomina claramente frente al mecanismo de deformación por flexión, mientras que en el segundo caso el mecanismo de deformación por flexión de la pared es mucho más relevante y condiciona el diseño del depósito.

En particular, se ha advertido la gran influencia que tiene la capacidad de giro de la unión pared-solera en el comportamiento del depósito. Cuando la unión es monolítica, el comportamiento del depósito está muy relacionado con el valor de las rigideces del terreno, de la solera y de la pared y es conveniente estudiar su comportamiento estructural con un modelo de análisis que contemple la interacción suelo-estructura. A modo de ejemplo, en la figura 1 se comparan los resultados obtenidos en un depósito de referencia, con unión monolítica en la base, para cuatro casos diferentes: consideración del análisis conjunto pared-solera-terreno (caso a); consideración del análisis conjunto pared-solera, suponiendo el terreno totalmente rígido (caso b); estudio aislado de la pared, suponiendo un empotramiento perfecto en la base (caso c); influencia de un aumento del espesor de la pared de $0,30 \mathrm{~m}$ a $0,45 \mathrm{~m}$ (caso d).

En cada uno de los cuatro casos citados, el pretensado se ha dimensionado de acuerdo con el criterio de compensar totalmente las tracciones anulares hidrostáticas. En el caso b, se observa la diferencia de comportamiento estructural del depósito en función del signo de la acción actuante: el análisis conjunto de un depósito con unión continua pared-solera suponiendo el terreno indeformable permite aproximar con gran precisión los esfuerzos debidos a la carga hidrostática, pero no es adecuado para el diseño del pretensado

El segundo grupo de estudios paramétricos tiene por objeto definir una ley de pretensado óptima, en base a unos criterios de optimación que conjugan aspectos estructurales con detalles constructivos. Como base de partida, se han estudiado diferentes funciones, algunas de ellas usuales y otras propuestas en este trabajo.

Los estudios persiguen, por un lado, la obtención de una función de pretensado que contrarreste la presión hidrostática y que dé lugar, en servicio, a un estado nulo de tracciones circunferenciales (denominada en este trabajo Función Hidrostática de Pretensado, F.H.P). Por otro lado, se persigue la definición de una función de pretensado queadicionalmente determine un estado de compresiones circunferenciales residuales mínimo $\sigma_{\text {res }}$ lo más uniforme posible (Función Uniforme de Pretensado, F.U.P), aspec- 
to éste último escasamente desarrollado en la literatura técnica. Esta compresión se plantea para evitar la fisuración vertical debida a otras acciones, tales como las reológicas y laacción térmica, que mermarían la estanquidad del depósito. truncada en la base, mientras que la F.U.P presenta una distribución uniforme en el tramo superior de la pared, y triangular en el tramo inferior. Se aprecia en ambas funciones que, mientras la definición en el tramo superior es parecida a las funciones usuales de proyecto (trapecial

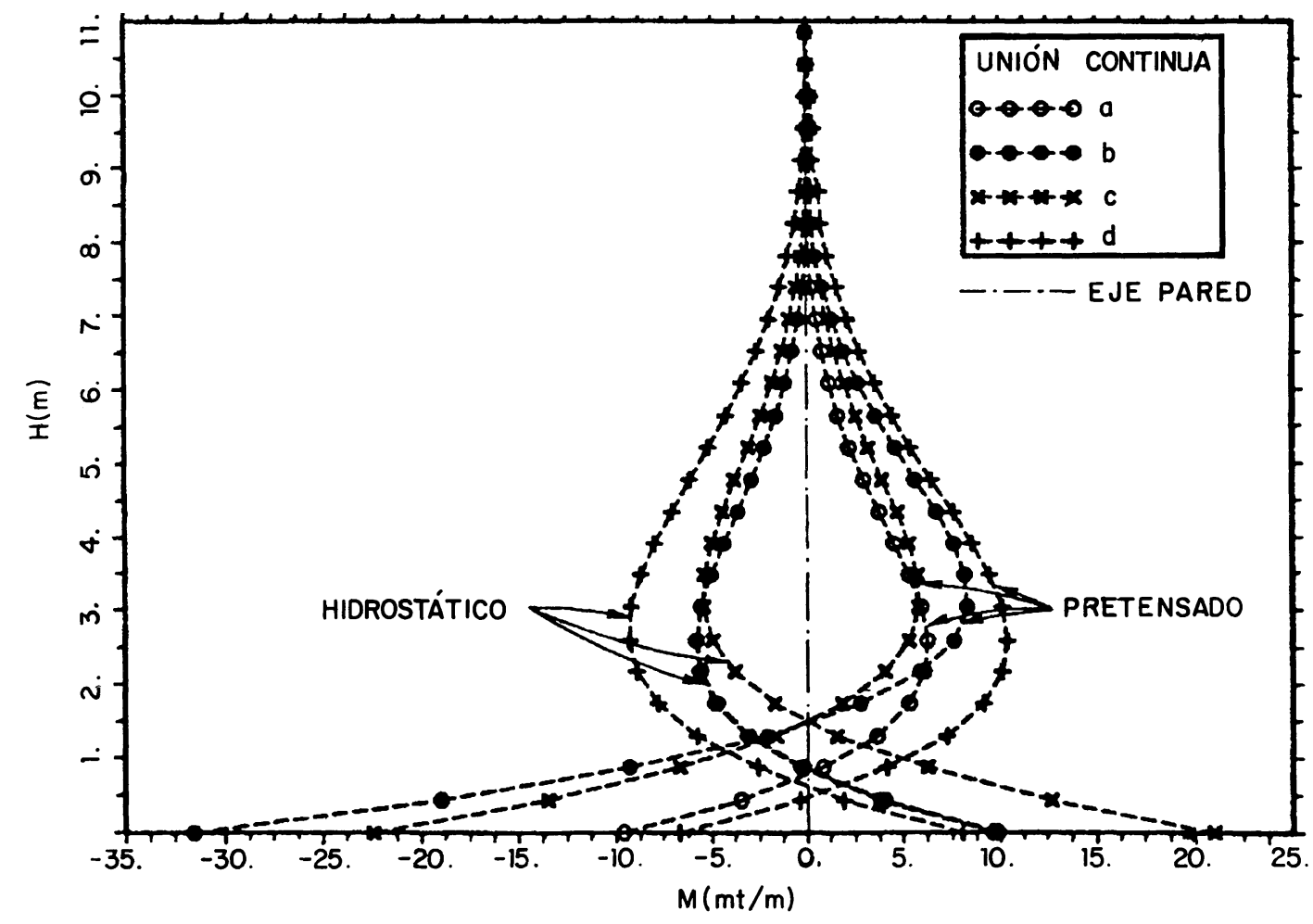

Depósito de referencia:

altura de agua $H_{w}=10,85 \mathrm{~m}$ espesor de solera $h_{s}=0,20 \mathrm{~m}$ espesor de pared $h_{p}=0,30 \mathrm{~m}$ rigidez del suelo $k_{s}=25 \mathrm{kp} / \mathrm{cm}^{3}$

Fig. 1.- Influencia en el comportamiento de la pared del depósito de la modelización de la unión entre la pared y la solera (a: análisis de interacción depósito-terreno; $b$ : análisis conjunto pared-solera, terreno totalmente rígido; $c$ : análisis de la pared aislada, suponiendo la base empotrada; d: influencia de un aumento del espesor de la pared).

La función óptima de pretensado obtenida en los estudios $(F . H . P+F . U . P)$ se caracteriza por minimizar la fuerza de pretensado necesaria y el esfuerzo cortante debido al pretensado en la base del depósito. Dicha función se ha definido para los tipos de unión entre la pared y la solera más usuales, contemplando tanto la fuerza de pretensado necesaria (con la que se obtiene el número de tendones) como el perfil de la función (con el que se determina la posición de cada uno de los tendones). Los resultados obtenidos se han comparado con otros criterios propuestos en la bibliografía, estudiándose la bondad de los mismos, y apreciándose ciertas lagunas en algunos criterios usuales en proyecto, tanto en lo referente a la estimación de la fuerza de pretensado como en lo que concierne a la distribución de los tendones.

En la figura 2 se ilustra la forma de las dos funciones propuestas. La F.H.P presenta una distribución trapecial para la $F . H . P$ y rectangular para la $F . U . P$ ), la definición en el tramo inferior es tal que intenta en lo posible alejar los tendones de la base, al ser una zona de difícil deformación anular por la coacción al movimiento de la unión.

A partir de los resultados obtenidos con los estudios paramétricos, se ha procedido a definir diferentes criterios de proyecto, que contemplan aspectos relacionados con el cálculo del depósito, el diseño del pretensado y la definición de la unión en la base.

En lo que concierne al cálculo de la pared del depósito, se proponen cuatro métodos generales. Los dos primeros son de aplicación para el caso de unión continua en la base, y se caracterizan por generalizar otros métodos simplificados usuales en la literatura técnica (método de Girkmann (1956) y método basado en calcular el depósito suponiendo la pared perfectamente empotradaen su base), introduciendo 
en éstos la influencia de las diferentes variables que definen la rigidez de la unión (espesores de la pared y de la solera, y la rigidez del terreno).

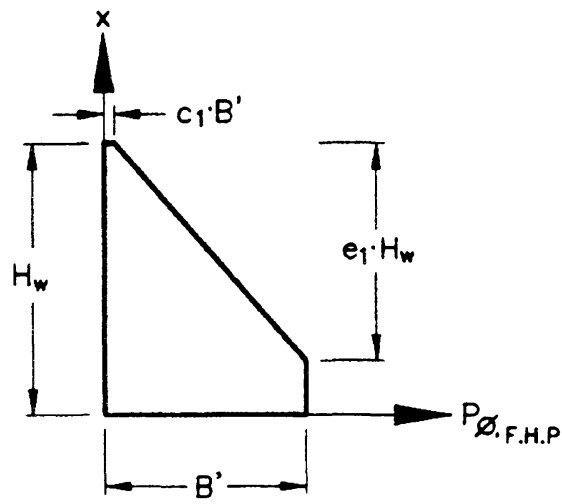

F.H.P

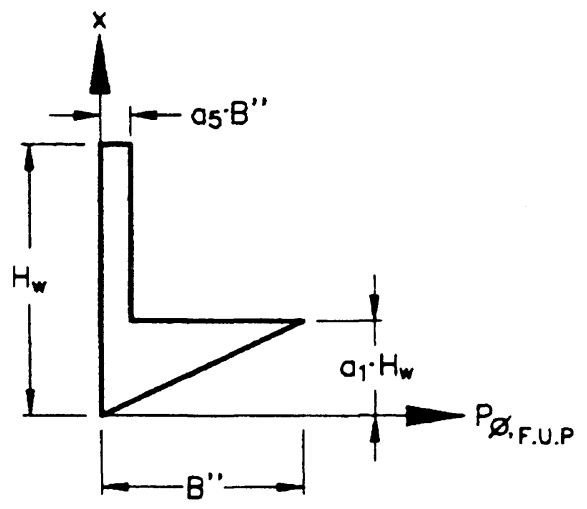

F.U.P
La figura 3 muestra, a modo de ejemplo, la relación entre el momento flector y el esfuerzo cortante en la base de la pared $M_{b}\left(h_{s}, k\right)$ y $Q_{b}\left(h_{s}, k_{s}\right)$ calculados considerando la
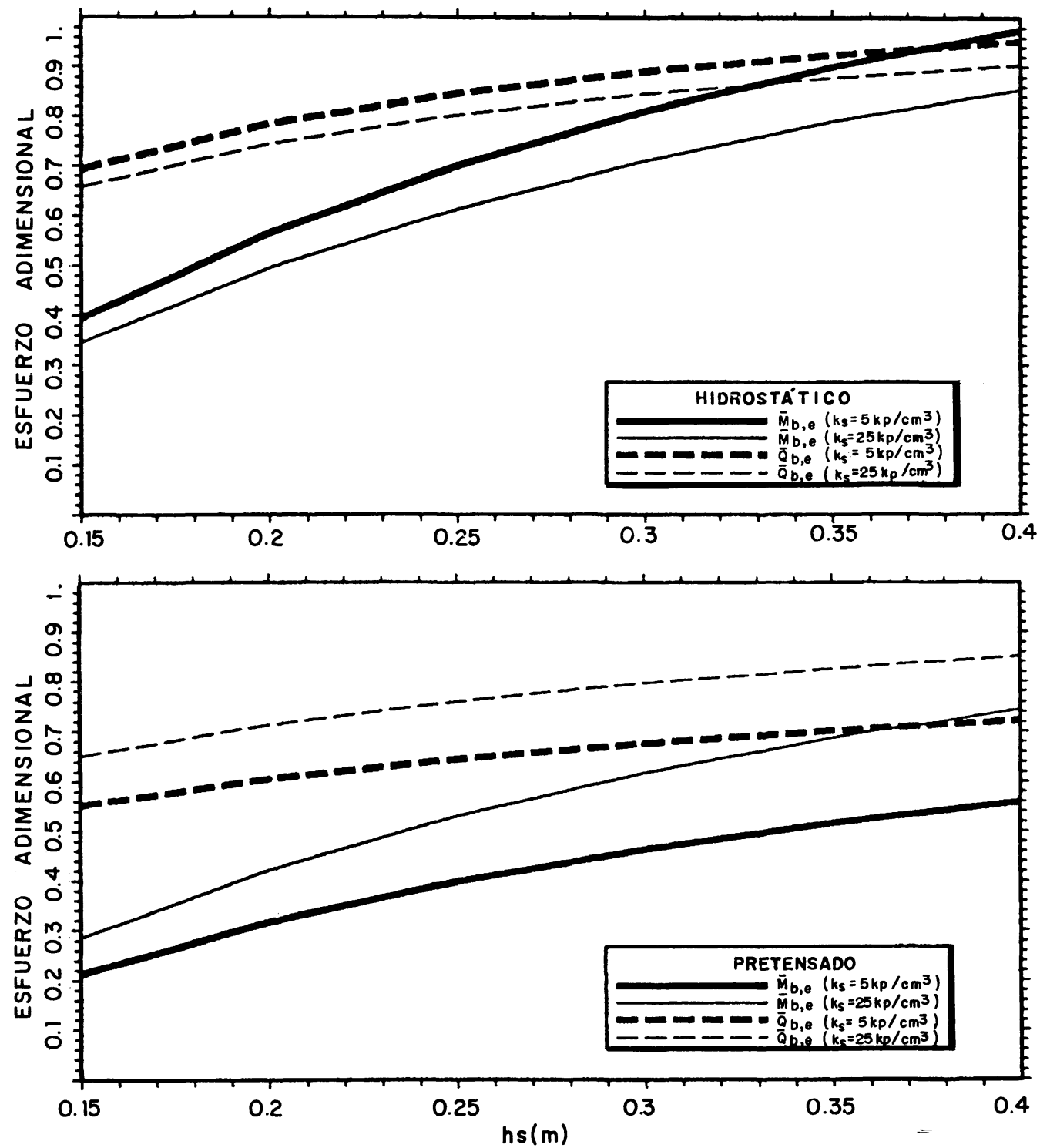

Fig. 3.- Generalización del método de cálculo de la pared suponiendo la pared perfectamente empotrada en la base. Esfuerzos adimensionales propuestos. 
interacción de la pared con la solera y el terreno, y los mismos esfuerzos obtenidos suponiendo la pared perfectamente empotrada en su base $M_{b, e}$ y $Q_{b, e}$ (a estas relaciones se las denomina esfuerzos adimensionales, $\bar{M}_{b, e}$ $=M_{b}\left(h_{s}, k\right) / M_{b, e}$ y $\left.\bar{Q}_{b, e}=Q_{b}\left(h_{s}, k\right) / Q_{b, e}\right)$, representando en la mencionada figura la variación de dichos esfuerzos adimensionales con la variación del espesor de la solera $h_{s}$, para dos tipos de terreno (módulos de balasto $k_{s}$ diferentes), y para dos estados de carga (hidrostática y pretensado).

En ella se puede apreciar que, en depósitos con espesores de solera bajos, las diferencias entre calcular los esfuerzos con un modelo de análisis que contemple la interacción suelo-estructura y calcularlos suponiendoun empotramiento perfecto en la base son muy importantes, especialmente en el caso de un depósito descansando sobre un terreno caracterizado con un módulo de balasto bajo y solicitado por el pretensado. Las diferencias observadas son ostensiblemente superiores en el momento flector que en el esfuerzo cortante en la base.

El tercer método propuesto para el cálculo de la pared se ha planteado para el caso de rótulafija en la base, mientras que el cuarto permite abordar el análisis de un depósito con un apoyo flexible en la base, definido por sus rigideces al desplazamiento radial y al giro.

Acerca del diseño del pretensado, se proponen distintos criterios para la definición de la función de pretensado, que tienen en cuenta el tipo de unión en la base. Estos criterios se centran tanto en la fuerza necesaria para mantener comprimida la pared con una tensión mínima circunferencial en la hipótesis de depósito lleno, como en la forma de la función.

En lo que se refiere a la fuerza de pretensado, ésta se define mediante una expresión matemática. La fuerza de la $F . H . P$ se expresa como

$$
P_{\phi, F . H . P}=\gamma_{w} R \frac{H_{w}^{2}}{2}
$$

siendo $\gamma_{w}$ el peso específico del fluido, $R$ el radio del depósito, y $H_{w}$ la altura libre de agua. Se aprecia que dicha fuerza corresponde a la integral de los axiles anulares hidrostáticos correspondientes a una lámina cilíndrica sin coacción alguna en sus contornos superior e inferior. Se observa, pues, que algunos criterios propuestos en la literatura técnica con los que se obtienen fuerzas menores pueden en ciertos casos no mantener comprimida la totalidad de la pared.

Por su parte, la fuerza de pretensado de la $F . U . P$ se expresa como

$$
P_{\phi, F . U . P}=\beta \sigma_{\text {res }} h_{p} H_{w}
$$

siendo $h_{p}$ el espesor de la pared, $\sigma_{\text {res }}$ la tensión residual de compresión mínima cuando el depósito está lleno, y $\beta$ un coeficiente de valor superior a la unidad que depende del tipo de unión en la base, de la esbeltez del depósito y de la altura inicial de la pared $H_{i n f}$ en la que se toleran tensiones de compresión inferiores a $\sigma_{\text {res }}$ (esta altura es de necesaria definición cuando la unión en la base es fija, dada la dificultad de comprimir anularmente el tramo de arranque de la pared, reduciéndose de esta manera razonablemente tanto la fuerza necesaria de pretensado como los esfuerzos de flexión). En la mencionada expresión se advierte que el término $\sigma_{r e s} h_{p} H_{w}$ corresponde a la integral de compresiones anulares en la pared de un depósito con los bordes superior e inferior libres y pretensada con una ley rectangular. Los valores propuestos en este trabajo para el parámetro $\beta$ son en algunos casos muy superiores a la unidad, por lo que se concluye que el criterio habitual de definir la fuerza de pretensado de la $F$. U.P con la expresión $\sigma_{r e s} h_{p} H_{w}$ es inseguro

En lo que se refiere a la forma de la ley de pretensado, se definen valores para los parámetros variables $\mathrm{c} 1$, e1, a1 y a5 (figura 2) de las funciones F.H.P y F.U.P, en base a parámetros de diseño como son el tipo de unión, el volumen y la esbeltez de la estructura, y el criterio de definición de la tensión de compresión residual $\sigma_{\text {res }}$

Por último, se proponen otros criterios de proyecto, encaminados a definir el tipo de unión en la base más adecuado, en función de la geometría del depósito (capacidad y relación $D / H_{w}$ ) y del diseño del pretensado. En primer lugar, se proponen criterios de definición de la unión en función de la tensión de compresión anular residual $\sigma_{\text {res }}$ y del volumen. En base a los criterios de análisis y de definición del pretensado propuestos, se ha observado que el rango de aplicación de la unión monolítica puede ampliarse hasta capacidades cercanas a $15.000 \mathrm{~m}^{3}$, cota superior a la observada en la literatura técnica. Con la articulación fija es posible llegar a capacidades ligeramente superiores a las correspondientes a la unión monolítica, siendo el apoyo flexible la solución más recomendable cuando la capacidad del depósito supera la barrera de los $15.000 \mathrm{~m}^{3}$, especialmente cuando debe definirse una tensión de compresión circunferencial mínima $\sigma_{\text {res }}$ superior a $5 \mathrm{kp} / \mathrm{cm}^{2}$ o debe asegurarse una compresión anular mínima en las proximidades del borde inferior de la pared (alturas $H_{i n f}$ bajas). Independientemente de la capacidad, este tipo de unión es asimismo el más recomendable cuando se requieren tensiones $\sigma_{\text {res }}$ superiores a $10 \mathrm{kp} / \mathrm{cm}^{2}$.

En segundo lugar, se proponen criterios de definición de la unión en la base en función de la esbeltez del depósito (relación $\mathrm{D} / \mathrm{H}_{\mathrm{w}}$ ), mediante la valoración de la eficacia del pretensado en la compresión anular de la pared (se define eficacia del pretensado a la relación entre la integral de axiles de compresión debidos al pretensado y la propia fuerza de pretensado). Este parámetro a su vez puede 
relacionarse con el coste de las armaduras activa y pasiva. Así, interesa obtener eficacias altas, dado que, para un mismo estado de compresión anular, se requiere menor cantidad de armadura activa, reduciéndose a su vez el comportamiento de flexión de la pared.

En función de la esbeltez $D / H_{w}$ del depósito, no todos los tipos de unión son compatibles con una eficacia mínima definida en proyecto. Para un mismo tipo de unión, la eficacia disminuye de manera aproximadamente lineal con el aumento de la relación $D / H_{w}$, mientras que, para una misma relación $D / H_{w}$, la eficacia es tanto mayor cuanto menor es la coacción a los movimientos del borde inferior de la pared. La eficacia también se ha relacionado con la altura inicial de la pared $H_{\text {inf }}$ en la que se permiten tensiones circunferenciales inferiores a la tensión de diseño $\sigma_{r e s}$

Los diferentes estudios realizados a lo largo de la tesis han permitido derivar un buen número de conclusiones, así como abrir la puerta a diferentes líneas futuras de investigación.

\section{Conclusiones}

Las conclusiones extraídas han sido numerosas, satisfaciéndose los objetivos propuestos. Dado el carácter del presente artículo (resumen de la tesis doctoral), se exponen a continuación únicamente aquellas conclusiones que pueden considerarse como más relevantes.

En primer lugar, puede afirmarse que el comportamiento estructural de un depósito cilíndrico depende en gran medida del tipo de unión en la base, distinguiéndose una gran diferencia entre un apoyo flexible y una unión con el desplazamiento radial impedido (rótula fija y unión monolítica). En este último caso, es aconsejable definir correctamente la rigidez al giro de la unión, mediante un modelo de análisis que contemple la interacción entre el suelo y la estructura.

En base a los múltiples estudios paramétricos realizados con el modelo de análisis desarrollado en este trabajo, se han formulado varios métodos sencillos para el cálculo de los esfuerzos de la pared, que generalizan otros métodos simplificados referidos en la literatura técnica. Los métodos propuestos en dicho trabajo tienen en cuenta tanto las características geométricas y mecánicas de la pared como el módulo de balasto del terreno y el espesor de la solera. Los resultados obtenidos han mostrado que, para los valores habituales de diseño de estos dos parámetros, se puede conseguir una reducción de los esfuerzos de flexión en la base tal que repercute en un ahorro en materiales (hormigón y acero) y en una generalización del uso de launión monolítica para depósitos con capacidades medias, tradicionalmente proyectados, a partir de cierto volumen, con otras tipologías.
En lo que se refiere a la función de pretensado, se han propuesto dos nuevas funciones, la Función Hidrostática de Pretensado (F.H.P) y la Función Uniforme de Pretensado (F.U.P). La primera tiene como misión compensar las tracciones anulares hidrostáticas en toda la pared y durante la vida útil de la estructura, mientras que la segunda tiene como misión generar adicionalmente una tensión de compresión circunferencial mínima en la pared. Se han planteado criterios para el cálculo de la fuerza de pretensado y para la definición de una distribución óptima de los tendones en la pared, atendiendo a criterios estructurales y económicos. Asimismo, se han comparado estos criterios con algunos procedimientos usuales en proyecto, observándose en algunos casos ciertas lagunas en estos últimos.

Por último, se han propuesto varios criterios de selección del tipo de unión más adecuada desde el punto de vista estructural, en función de la geometría del depósito y del diseño del pretensado. En base a los criterios de análisis y de definición del pretensado propuestos, se ha observado que el rango de aplicación de la unión monolítica puede ampliarse hasta capacidades cercanas a los $15.000 \mathrm{~m}^{3}$, siendo aconsejable el apoyo flexible para capacidades superiores o para aquellos casos en los que se requiere mantener comprimida la pared con tensiones en servicio superiores a los $10 \mathrm{kp} / \mathrm{cm}^{2}$.

\section{Bibliografía}

AGUADO, A., MARÍ, A.R. Y PEÑALVA, C.: "Proyecto Tipo de Depósitos Cilindricos Para Agua de Hormigón Proyectado con Armaduras Postesas", Convenio n ${ }^{\circ}$. 619/TC 197 Corporación Metropolitana de Barcelona-U.P.C., Barcelona, enero 1985.

AGUADO, A., MARÍ, A.R., MIRAMBELL, E. Y BOIXEREU, E.: "Orden de Tesado en Depósitos de Hormigón Pretensado de Pequeña Capacidad", Hormigón y Acero n.173, $4^{\circ}$ trimestre 1989, pp. 103-111.

BRONDUM-NIELSEN, T.: "Prestressed Tanks", ACI Journal, vol.82, $\mathrm{n}^{\circ}$ 4, July-August 1985, pp. 500-509.

DEL POZO, F.: "Depósitos Cilindricos Circulares", Laboratorio Central de Ensayo de Materiales de Construcción, Publicación $\mathrm{n}^{\circ} 195$, Madrid 1967, 62 págs.

GHALI, A.: "CircularStorageTanks and Silos", E.\& F.N. Spon Ltd, London 1979, 210 págs.

GIRKMANN, K.: "Flachentragwerke", Ed. Springer, 4th ed., Vienna 1956, pp. 448-458.

LLOMBART, J.A. Y ANTÓN, V.: "Depósitos Cilindricos Pretensados. Consideraciones sobre el Proyecto", Hormigón y Acero ${ }^{\circ} 157,1985$, pp. 141-156.

TIMOSHENKO, S.P. Y WOINOWSKY KRRIEGER, S.: "Theory of Plates and Shells", Mc. Graw-Hill Book Co., Singapore 1959, 580 pág. 
VAN BREUGEL, K.: "Problems Associated with Wall-to-Base Connections", Concrete Society Conference on Design, Construction and Maintenance of Concrete Storage Structures, Newcastle Upon Tyne, December 1984, pp.51-65.

VILARDELL, J.M.: "Anàlisi Estructural i Criteris de Disseny de Dipòsits Cilindrics de Formigó Pretensat", Tesina de Especialidad, E.T.S. Ingenieros de Caminos, Canales y Puertos, U.P.C., Barcelona, junio 1990
VILARDELL, J.M., AGUADO, A. Y MIRAMBELL, E.: "Depósitos cilíndricos de hormigón pretensado: una nueva visión de cálculo", Hormigón y Acero, n 191, mayo 1994, pp. 40-43.

VILARDELL, J.M.: “Análisis estructural y criterios de diseño dedepósitos cilindricos de hormigón pretensado", Tesis Doctoral, Departamento de la Ingeniería de la Construcción, U.P.C, julio 1994,577 pàgs.

\section{publicaciones del IETCC/CSIC}

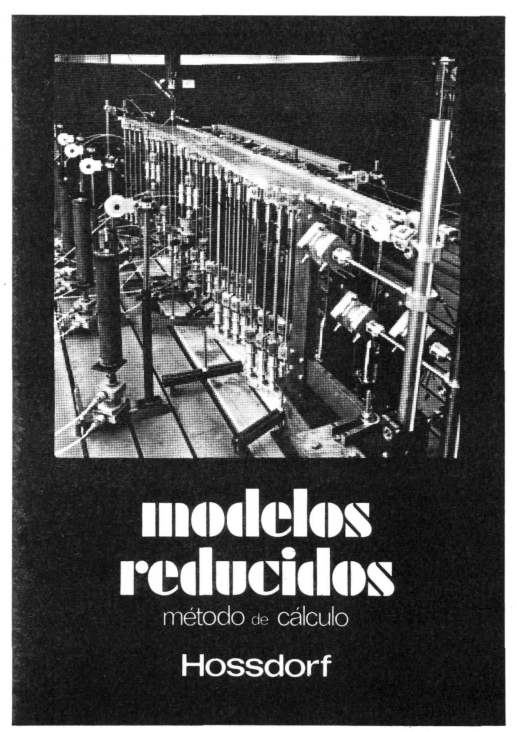

Modelos reducidos. Método de cálculo

H. Hossdorf, Ingeniero Civil

La técnica de los ensayos en modelos reducidos de estructuras sufre hoy día una decisiva metamorfosis. hasta hace poco era un medio más bien de artesaa academicos teorizantes Dara comprender el comportaque que se acudió las más de las veces, como a un ultimo remedio debido a sus indiscutibles insuficiencias. Sin embargo, en poco tiempo y gracias a su conexion con los, endenadores digitales, se ha transcormado en un instricione que no puede querar

Un volumen encuadernado en cartoné plastificado con lomo de tela, de $17 \times 24 \mathrm{~cm}$, compuesto de 250 páginas, 158 figuras y fotografias.

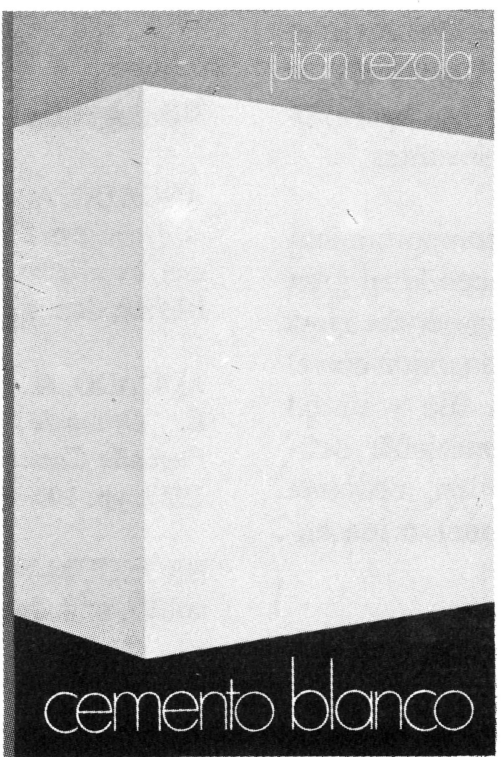

\section{Cemento blanco}

Julián Rezola

Ingeniero Quimico Dipl. I. Q. S.

Sabido es que existe una extensa y documentada bibliografía sobre el cemento gris: en cambio, no puede decirse lo mismo acerca del cemento portland blanco, ya que los escritos existentes se refieren tan sólo a algunas peculiaridades que le distinguen de aquél.

El autor nos ofrece sus profundos conocimientos y su larga experiencia tanto en laboratorio como en fabricación.

La parte descriptiva del libro se complementa con gráficos, diagramas y fotografías de gran utilidad, destinados a conseguir la aplicación apropiada de este aglomerante.

Un volumen encuadernado en cartoné policerado, de $17,4 \times 24,3 \mathrm{~cm}$, compuesto de 395 páginas, numerosas figuras, tablas $y$ ábacos.

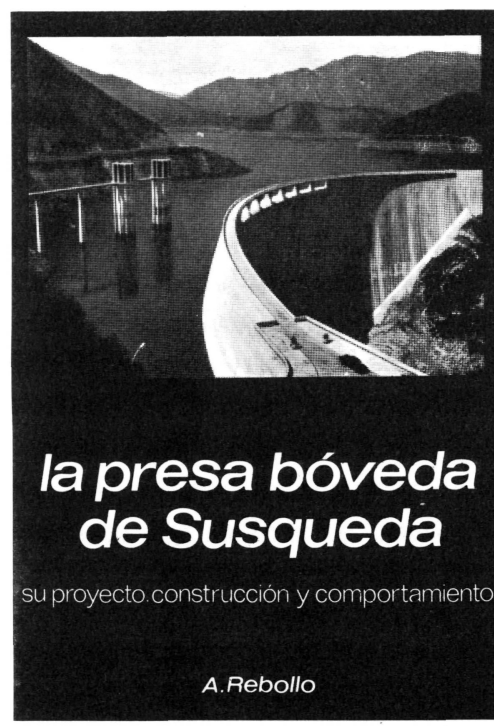

La presa bóveda de Susqueda

A. Rebollo,

Dr. Ingeniero de Caminos

El esfuerzo del constructor de presas se sitúa, por su pretensión de perennidad, a contracorriente de las tendencias de la civilizacion actual, caracterizada por to fungible. Pueden evocarse las 10.000 grandes. presas en funcionamiento o en construcción que están envejeciendo y reclaman los cuidados gerontologicos para mantener y perfeccionar su servicio y garantizar su inalienable pretensión de perennidad. En la medida en que todas nuevas obras, grandes o pequeñas, son portadoras de riesgos ecologicos $y$, a veces, catastróficos, que aumentan con el envejecimiento, la gerontologia de las presas es todo un emplazo. La acción adelantada de Arturo Rebollo en este terreno marca un camino

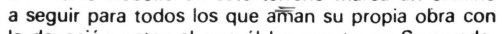
la devoción paternal que él ha puesto en Susqueda.

Un volumen encuadernado en cartoné plastificado con lomo de tela, de $18 \times 24,5 \mathrm{~cm}$, compuesto de 408 páginas, 330 figuras y fotografias y 39 tablas. 